YCTP-P8-92

Revised March 1992

\title{
SOLAR NEUTRINO DATA AND ITS IMPLICATIONS
}

\author{
Evalyn Gates", Lawrence Krauss [2] and Martin White \\ Center for Theoretical Physics, Sloane Laboratory \\ Yale University, New Haven, CT 06511, USA
}

\begin{abstract}
The complete and concurrent Homestake and Kamiokande solar neutrino data sets (including backgrounds), when compared to detailed model predictions, provide no unambiguous indication of the solution to the solar neutrino problem. All neutrino-based solutions, including time-varying models, provide reasonable fits to both the 3 year concurrent data and the full 20 year data set. A simple constant $B$ neutrino flux reduction is ruled out at greater than the $4 \sigma$ level for both data sets. While such a flux reduction provides a marginal fit to the unweighted averages of the concurrent data, it does not provide a good fit to the average of the full 20 year sample. Gallium experiments may not be able to distinguish between the currently allowed neutrino-based possibilities.
\end{abstract}

\footnotetext{
${ }^{1}$ Supported in part by the DOE. Address after October 1: Department of Physics, University of Chicago.

${ }^{2}$ Also Department of Astronomy. Research supported in part by the DOE, the NSF and the TNRLC. Bitnet: KRAUSS@YALEHEP

${ }^{3}$ Research supported in part by a grant from the TNRLC. Address after September 1: Center for Particle Astrophysics, University of California, Berkeley.
} 
Perhaps at no time in the past 20 years has there been more interest in the solar neutrino problem than at the present moment. The apparent deficit of high energy solar neutrinos observed by the Homestake Solar Neutrino Detector over two decades [1] has now been confirmed by the Kamiokande large underground water Cerenkov detector [2]. Gallium detectors are beginning to come online and the SAGE group has recently published their first results [22] which seem to indicate a neutrino deficit which cannot be explained by solar physics (for a brief discussion see 21]). Other detectors are approved or are in the planning stages, and there is hope that a solution to the solar neutrino problem may be at hand. At the same time a growing number of theoretical neutrino-based "solutions" have been proposed. Heading the list appears to be the MSW solution [5, 6], which, in a restricted but reasonable range of neutrino masses and mixing angles, allows significant reduction in the neutrino signal to be observed. An alternative solution involves a large neutrino magnetic moment, either diagonal or transitional, which causes neutrinos to oscillate into "sterile" partners while traversing the magnetic field of the sun [7, 8]. While this seems less theoretically compelling, especially in view of the large neutrino magnetic moments required, it has the distinct advantage of allowing not only the neutrino signal to be time varying over the solar cycle, but also allows for a different time variation to be observed in different detectors! 9].

It may seem a priori that the simplest solution of the original $\mathrm{Cl}$ solar neutrino problem resides in the solar model itself, namely that fewer high energy neutrinos are created in the sun than the standard solar model suggests. It is important to determine if this possibility can be ruled out, although it seems increasingly difficult to accommodate, especially in light of the new SAGE results [22]. Also, astrophysical mechanisms which reduce the high energy neutrino flux are now not supported by any other solar measurements (most importantly the p-mode fine structure [23, 1]). Meanwhile, several studies incorporating recent $\mathrm{Cl}$ data into the 20 year observations provide 
very tempting, if not compelling, evidence of time variations in the $\mathrm{Cl}$ signal [11, 17, 13] which may be correlated, in some yet to be determined way, with the solar cycle. On the other hand, the Kamiokande data appears naively to show no such time variation. The presence, for the first time, of two different data sets for the solar neutrino signal should allow a number of finer tests of solar neutrino models to be made (see e.g. [2]). Surprisingly, however, rarely have all the data been used. For example, analyses have been performed comparing the Homestake 20 year "average" signal with the averaged Kamiokande 3 year signal. It is not clear that such a procedure is correct. Until we have a better idea of what is at the root of the solar neutrino problem, we can make no a priori claims about what the Kamiokande signal would have been if the detector had also taken data during the 20 years Homestake was operational, especially given the apparent variations in the $\mathrm{Cl}$ data during this period. All of the data points from both experiments should be exploited, and the error bars examined. For guidance on how to treat the entire data sets one can first analyse the data during the period in which the two detectors were both running concurrently. It is only during this time that we have a direct independent check on the $\mathrm{Cl}$ data, and can check for consistency between the data sets. One might then be guided on how to use all the data to test various hypotheses. This is the spirit of the following work. We have utilized the entire Homestake and Kamiokande data sets, concentrating first on the concurrent data sets and then on all the data, in order to investigate the range of models which may or may not fit the data. We have carried out extensive numerical model calculations, in which neutrinos are propagated, with complete phase information, through much of the sun, in order to estimate the flux in various neutrino species at the earth's surface. We have also used realistic models of detector sensitivity in order to turn fluxes into detection rates.

We emphasize that using the concurrent Kamiokande data to "check" the $\mathrm{Cl}$ data is important beyond the strict question of whether or not any solar 
cycle time variation exists. It allows us to understand how best to treat the full 20 year $\mathrm{Cl}$ data to explore solutions to the solar neutrino problem. We display in figure 1 (a) and (b) the two full data sets, and the concurrent data sets. Both data sets are normalized to the Standard Solar model [1] predictions (see section (3)).

While quoted averages of the two data sets appear at first sight to differ, when the full data sets are displayed this issue is less clear. The $\mathrm{Cl}$ signal clearly has much more jitter, with several apparently anomalously low points, but aside from this one might not be surprised if told that all data came from a single detector. This may suggest that a simple, energy independent deficit of $\mathrm{B}$ neutrinos could be consistent with all of the data. To properly explore this possibility, as well as the possibility that the solar neutrino deficit is neutrino related, a more quantitative approach is required.

\section{Neutrino flux at the earth}

The neutrino spectrum predicted by the Standard Solar Model (SSM) is described in detail by Bahcall in [1]. The dominant neutrino flux, that due to the pp reaction in the sun, with energies less than $0.42 \mathrm{MeV}$, is unobservable in both the $\mathrm{Cl}$ and Kamiokande detectors, due to their thresholds. The component of the flux which gives the dominant contribution to the $\mathrm{Cl}$ signal, and the entire contribution to Kamiokande is the high energy ${ }^{8} B$ continuous spectrum $\left({ }^{8} B \rightarrow^{7} B e^{*}+e^{+}+\nu_{e}\right)$, with neutrino energies up to $15 \mathrm{MeV}$ and a total predicted flux at the earth of $(5.8 \pm 2.2) \times 10^{6} \mathrm{~cm}^{-2} s^{-1}$ ( " $3 \sigma$ " theoretical error). The only other component of the neutrino spectrum contributing to the $\mathrm{Cl}$ signal at greater than the $5 \%$ level are the $\mathrm{Be}$ neutrinos $\left({ }^{7} \mathrm{Be}+e^{-} \rightarrow^{7}\right.$ $\left.L i+\nu_{e}\right)$, with fixed energy $0.862 \mathrm{MeV}$ and a predicted flux of $4.7 \pm .7(3 \sigma) \times$ $10^{9} \mathrm{~cm}^{-2} \mathrm{~s}^{-1}$.

There are two ways one might expect to alter these predicted fluxes. First one might lower the overall flux by a fixed amount by postulating some new solar physics. For example, if the core temperature is lowered compared 
to the SSM, the B signal can be significantly reduced (such a temperature reduction is the aim of many non-standard solar models, e.g. see [1]).

We have incorporated these possibilities in our analysis by treating the net B flux as a free parameter in one set of runs, and examining the goodness of fit with the combined data sets as this parameter is varied compared to the SSM. While this is a very simplistic "non-standard solar model" we can use it to perform straightforward statistical tests of how well the data is fit by models aiming at such a B flux reduction.

The other possibility is that the origin of the solar neutrino problem lies in the properties of neutrinos themselves. If neutrinos have non-zero mass eigenstates which do not coincide with weak eigenstates, neutrino propagation will lead to oscillations between the different weak states, namely between electron, muon, and tau neutrinos. Since the $\mathrm{Cl}$ detector is sensitive only to electron neutrinos, while the Kamiokande water detector is sensitive predominantly to electron neutrinos, such oscillations could have the possibility of reducing the observed signal in both detectors. Moreover, the presence of matter can enhance the oscillations between neutrino species [5] due to the presence of level crossings which occur as the background electron density varies. If one supplements neutrino masses with large magnetic moments, which in general need not be diagonal in the weak basis, then another possibility arises. Magnetic fields in the sun could cause oscillations between left and right handed neutrino states, with or without induced level crossings [7]. In general, left-right mixing can allow neutrino states to oscillate into antineutrino states, unlike the pure MSW mechanism [8]. In any case, as long as the right handed states have suppressed interaction rates in the detectors, this can reduce the observed neutrino signal. Moreover, it allows for a possible correlation with the solar cycle, although the required neutrino magnetic moments, at least for currently envisaged magnetic field strengths in the sun, are large enough to cause other potential astrophysical problems [14]. Finally, in the most general case, both effects may be operational with 
the different factors dominating in different regimes of mass, mixing angle, and magnetic field space [8]. This allows independent time variations to be observed in the two detectors [9], and it is this general case which we shall consider here.

We followed explicitly the propagation of neutrinos through the sun by numerically integrating the Hamiltonian evolution equation for neutrinos through matter for a two generation model with Majorana-type transition magnetic moment and off diagonal mass terms [8, 9, 15, 16].

$$
i \frac{d}{d t}\left[\begin{array}{c}
\nu_{e} \\
\nu_{\mu} \\
\bar{\nu}_{e} \\
\bar{\nu}_{\mu}
\end{array}\right]=H\left[\begin{array}{c}
\nu_{e} \\
\nu_{\mu} \\
\bar{\nu}_{e} \\
\bar{\nu}_{\mu}
\end{array}\right]
$$

The Hamiltonian for the system [8] is given by

$$
H=\left[\begin{array}{cccc}
a_{e} & \frac{\Delta m^{2}}{4 E_{\nu}} \sin 2 \theta & 0 & \mu B \\
\frac{\Delta m^{2}}{4 E_{\nu}} \sin 2 \theta & \frac{\Delta m^{2}}{2 E_{\nu}} \cos 2 \theta+a_{\mu} & -\mu B & 0 \\
0 & -\mu B & -a_{e} & \frac{\Delta m^{2}}{4 E_{\nu}} \sin 2 \theta \\
\mu B & 0 & \frac{\Delta m^{2}}{4 E_{\nu}} \sin 2 \theta & \frac{\Delta m^{2}}{2 E_{\nu}} \cos 2 \theta-a_{\mu}
\end{array}\right]
$$

where B is the magnetic field, $a_{e}=G_{F}\left(2 N_{e}-N_{n}\right) / \sqrt{2}$ and $a_{\mu}=G_{F}\left(-N_{n}\right) / \sqrt{2}$ with $N_{e}, N_{n}$ the electron and neutron densities as a function of radius in the solar interior. We used the following fit to the electron and neutron densities in the standard model sun [1]

$$
\begin{aligned}
& N_{e}= \begin{cases}2.45 \times 10^{26} \exp (-10.54 x) & 0.2<x<1 \\
6 \times 10^{25}[1-10 x / 3] / \mathrm{cm}^{3} & 0.1<x<0.2\end{cases} \\
& N_{n}= \begin{cases}2.45 \times 10^{26} \exp (-10.54 x) & 0.2<x<1 \\
2 \times 10^{25}[1-21 x / 5] / \mathrm{cm}^{3} & 0.1<x<0.2\end{cases}
\end{aligned}
$$

where $x=r / R_{\odot}$. 
The free parameters in the calculation are the neutrino energy E, masssquared difference $\Delta m^{2}$, vacuum mixing angle $\sin ^{2}(2 \theta)$ and Zeeman energy $\mu B$ : the product of the transition magnetic moment and solar magnetic field. For reasons of simplicity we assumed this to be uniform over the radiation and convection zones in the sun, falling sharply to zero at the exterior.

The evolution in the interior was performed using a Runge-Kutta algorithm with adaptive step size control [15] in double precision arithmetic. On order of $10^{5}$ steps were taken for the higher mass gaps, the neutrinos being evolved from just before the resonance [16]

$$
\left.\sqrt{2} G_{F} N_{e}\right|_{\text {res }}=\frac{\Delta m^{2}}{2 E} \cos (2 \theta)
$$

to the edge of the Sun.

In the exterior of the sun, where the magnetic field is assumed to be zero, the neutrino and anti-neutrino sectors decouple and the vacuum oscillations can be computed using standard analytic formulae (see [16]). Since each detector signal averages over a period of at least 2 months (though not necessarily weighting times evenly) we modelled the motion of the Earth in a simple way by averaging the vacuum oscillations over an Earth-Sun distance of $d(1-e / 2)$ to $d(1+e / 2)$, where the semi-major axis is $d=1.496 \times 10^{8} \mathrm{~km}$ and the eccentricity of the Earth's orbit is $e=0.0167$. This corresponds to the variation in the Earth-Sun distance over 3 months.

The general form of the propagation matrix for neutrinos allows for the conversion of electron neutrinos into muon neutrinos and also into muon and electron antineutrinos. The latter conversion can occur in two steps, either by a magnetic moment induced oscillation followed by an MSW type oscillation, or the reverse. Assuming initially electron neutrinos are emitted, the probability $P_{i}$ of finding each of the 4 species at the Earth was computed for a grid of the 4 parameters. For the continuum spectra we calculated the probabilities for 30 energies ranging from $0.5 \mathrm{MeV}$ to $15 \mathrm{MeV}$ in $0.5 \mathrm{MeV}$ steps, and we also calculated the probabilities at $0.862 \mathrm{MeV}$ and $1.442 \mathrm{MeV}$ 
corresponding to the ${ }^{7} \mathrm{Be}$ and pep neutrino lines respectively. The mass gap, $\Delta m^{2}$, ranged from $10^{-5} \mathrm{eV}^{2}$ to $10^{-8} \mathrm{eV}^{2}$; for higher mass gaps the Zeeman energy plays no role and pure MSW/vacuum mixing, results. This case has been well studied and the higher mass gaps in the so called "adiabatic regime" may already be ruled out by experiment [2]. The vacuum mixing angle, $\sin ^{2}(2 \theta)$, ranged from 0.01 to 1.00 and the Zeeman energies, $\mu B$, from 0 to $5 \times$ $10^{-10} \mu \mathrm{BkG}$. The best limit on neutrino transition moments is astrophysical, coming from the luminosity of red giant stars before and after the He flash [14],

$$
\mu<3 \times 10^{-12} \mu_{B}(3 \sigma)
$$

and the best lab limits (from $\bar{\nu}_{e}-e$ scattering) are [20]

$$
\left|\kappa_{e}\right|<4 \times 10^{-10},\left|\kappa_{\mu}\right|<10^{-9} ; \quad \mu_{i}=\kappa_{i} \mu_{B}
$$

so the larger Zeeman energies require enormous fields in the solar interior.

The expected event rates in the detectors were calculated by convolving known neutrino cross sections with published detector efficiencies [1, 17, 18].

\section{The Data}

The 90 Homestake data points between the years 1970 and 1991 were obtained with about 2 months of integration time per point. The time shown for each Homestake data point in figure 1 is the mean time of production of the radioactive Argon atoms (see [1] for a description). For each point the experiment reported an upper limit on the production rate, a lower limit on the rate, and the mean value of the rate, all determined by a maximum likelihood fit to the data [4]. The errors about the mean were generally symmetric, except in the case where the lower limit would have become negative, in which case the reported error bars were quoted as half the difference between the upper limit and zero, and were thus sometimes artificially small. This suppression of the errors would artificially increase the weighting of these points in any fit to the data. In order to remove this effect, we utilized 
fully symmetric error bars on all points. The size of $1 \sigma$ error bars was fixed to be the difference between the reported upper limit and the mean value for each point. It has been calculated that $.08 \pm .03$ argon atoms/day are produced [1] by the (muon induced) background. In determining the average Homestake signal it is appropriate to subtract this background after the average Ar rate has been computed from the total data set, and add errors in quadrature. When performing a point by point fit of theory to the data, however, it is appropriate to subtract this background from each data point and add its uncertainty to the rate uncertainty for each point in quadrature. ff Figure 1 displays the values divided by the standard solar model (SSM) predicted rate. Because of the unusually small errors on many of the points with small rates, the treatment of errors in the Homestake experiment has been an issue of some debate. In particular the "error" determined by the maximum likelihood fit is not a Gaussian $1 \sigma$ error for points with small numbers of counts $(N \leq 5)$ and the use of a $\chi^{2}$ analysis will not weight these points correctly (see e.g. [3]). To consider the effect of this, for the analysis of the non-standard solar models and the MSW neutrino model we also used the method of [3] to analyze the Homestake data, while still using $\chi^{2}$ for the Kamiokande data.

The Kamiokande data is more straightforward. Over the period 19871990, five data points have been obtained, based on real time measurements of the directional solar neutrino signal, averaged over a period of several months. These data points, along with errors, were presented as a fraction of the rate predicted by the SSM [2], and, as shown in figure 1, were used

\footnotetext{
${ }^{4}$ The average rate (which converts to $.26 \pm .04 \mathrm{SSM}$ ) quoted by the Homestake group comes from a maximum likelihood fit of $\mathrm{N}=61$ runs to a constant background plus 1 decaying species. The division of the counts into (counter) background and signal is different if the runs are analysed separately or collectively, the (counter) background in a run by run analysis being quite variable. We calculate our average rate as the average of the values quoted per run, for $\mathrm{N}=90$ runs. Note that .26 is bracketed by our weighted and unweighted values. The larger error, .04, is consistent with the smaller number of runs analysed by the Homestake group.
} 
directly in this analysis.

Finally, we decided not to additionally weight the Kamiokande and $\mathrm{Cl}$ data points in terms of the length of the measuring interval associated with each point. In the first place, longer runs in the $\mathrm{Cl}$ experiment do not mean more data. Because the produced Ar atoms decay with a 35 day half life they will eventually reach an equilibrium abundance after several months exposure. Secondly, the small error bars on the Kamiokande data points presumably reflect the longer exposure times for each point in this experiment, and thus measuring time will in this case naturally be taken into account in any weighting by errors of the data.

\section{Analysis}

In an effort to determine how the current solar neutrino data constrains the various possible models discussed in (2) we compared the predicted signals in both detectors to the data by means of a $\chi^{2}$ goodness-of-fit procedure. For each model we computed the predicted signal over a range of model pa-

rameters, and for each combination calculated the value of $\chi^{2}$ for the signal compared to the data. We then examined the parameter space for $\chi^{2}$ values corresponding to confidence levels of $68 \%$ and $95 \%$.

The different models we considered are:

(a) Non-standard solar model: B flux reduction

(b) Non-standard solar model: $(\mathrm{B}+\mathrm{Be})$ flux reduction

(c) Neutrino Mass model (no magnetic moments-constant flux)

(d) Neutrino Mass model (with magnetic moments-variable flux)

In case (c), each combination of the neutrino mass-squared difference and vacuum mixing angle produced a constant fit to each of the detector signals. In case $(\mathrm{d})$ in addition to these parameters, the quantity $\mu B$ was assumed to 
have the form $\mu B=A+C f(t)$. In this case $f(t)$ was set to either $\cos (\phi+k t)$, where $\phi$ and $k$ were determined from sunspot data, or to a sawtooth function of unit amplitude with a net period equal to the solar cycle and the position of the cusp given by time $\tau$. This latter model was chosen based on an earlier suggestion by Bahcall and Press [11] that the neutrino time variation could be well described by such a function. We considered $\tau=8.05$ years, based on their fit to the Ar data, and $\tau=6.65$ years based on their fit to sunspot data. Thus in case $(\mathrm{d})$ there are two additional parameters, A and $\mathrm{C}$, involved. The translation of $\chi^{2}$ values into confidence levels depends upon number of degrees of freedom. In determining the goodness of fit of models $(a, b, c)$ with various sets of parameters, the number of degrees of freedom was set equal to the number of data points, since the model predictions are fixed once the parameters are fixed, and no parameter in this test is minimized to fit the data. In model (d) the number of degrees of freedom was reduced by 2 since $\mathrm{A}$ and $\mathrm{C}$ were fit to the data before goodness of fit was evaluated.

Our results are displayed in Tables 2, 3 and figures $2-7$. The tables list the "best fit" (i.e. smallest $\chi^{2}$ ) model parameters along with degrees of freedom (df)色 for fits to:

(i) the concurrent 3 year data, and the averaged 3 year data,

(ii) the complete 20 year data set, and the averaged 20 year data

We do not place much significance on the actual value of the best fit parameters, rather we would emphasize the regions in $\Delta m^{2}-\sin ^{2}(2 \theta)$ space for which the model fits the data at a given confidence level.

Let us review the fits to each of the data sets in turn.

(i) Concurrent data set: In spite the apparent similarity of the two signals during this period, the simplest apparent resolution of the solar neutrino

\footnotetext{
${ }^{5}$ Note the number of degrees of freedom to be used for a goodness-of-fit and the number quoted for a "best fit" are not the same, the latter being smaller by the number of parameters varied in the fit.
} 
problem, that obtained by reducing the B neutrino flux alone, is ruled out at greater than the $4 \sigma$ level based on a comparison with the weighted data points (including a Be reduction by the same amount allows a fit at the $3 \sigma$ level). This discrepancy is because the small error bars on the low Homestake points heavily skew any fit. The mean value of the Homestake data during this period rises from 25 to .36 of the SSM prediction if each point is equally weighted and the fit to a non-standard solar model improves dramatically. In this case, if the SSM B flux is reduced by a constant factor, the fit to the unweighted averages is acceptable over a small range at the $99 \%$ confidence level. Whether or not to ignore the heavy weighting of the apparent anomalously low $\mathrm{Cl}$ data points therefore becomes an important issue if one is to claim non-standard solar models are ruled out by the combination of $\mathrm{Cl}$ and Kamiokande data, at least during the period in which the data was taken concurrently. If the procedure of [3] is used the non-standard solar model just fits the concurrent data at the $99 \%$ confidence level 0 , with the favored boron flux reduction at $37 \%$ of the SSM.

If no model fit the complete fully weighted concurrent data sets, this would provide strong evidence in favor of the assumption that the jitter in the $\mathrm{Cl}$ signal precludes its use directly in constraining models, and might provide motivation for ignoring the quoted error bars on the data. As can be seen, however, all the models with neutrino masses, including those with a time variability, provide reasonable fits to the data (at 95\% confidence level). The range of fit of the MSW model to this concurrent sample is shown in figure 2 (a), along with the claimed fit to the 20 year averaged data by Bahcall and Bethe [6] (solid line). We see that the Bahcall and Bethe line passes through the arm of the $95 \%$ confidence level region. If the unweighted averages of the Homestake data sets and the Kamiokande average rate are

\footnotetext{
${ }^{6}$ The procedure of [3] makes use of the likelihood ratio test in which the test statistic is $\chi^{2}$ distributed in the limit of a large number of data points. In applying this test to the concurrent Homestake data we should bear in mind that there are only 20 data points.
} 
compared to the MSW prediction, the allowed regions are shown in figure 2 (b). Notice that the fit to the unweighted average is good at the $68 \%$ level over a range of parameters and the $68 \%$ region coincides with the Bahcall and Bethe best fit line. An almost identical region is obtained for the fit to the weighted averages of the data, suggesting the poorer fit in the case of the individual points is due to "jitter" in the data. If the analysis is done using the method of [3] the MSW model still fits, though the goodness-of-fit is slightly worse than for the case of the straightforward $\chi^{2}$ fit.

We now switch to the time dependent fits, involving a non-zero transition magnetic moment. The "best fit" magnetic field peak Zeeman energy has a value of $4.6 \times 10^{-10} \mu B \mathrm{kG}$ for the cosine and $4-5 \times 10^{-10} \mu B \mathrm{kG}$ for the sawtooth fits, which are essentially as good as the MSW fits. Because the 20 year data provides more compelling evidence of time variability, we also investigated the goodness of fit of the 20 year "best fit" parameters to the 3 year concurrent set in the time varying models. The "best fit" values differ somewhat from the best fit to the 3 year data, but they are still comparably good. This indicates that there is no evidence from the concurrent data against the same time variation inferred from the 20 year $\mathrm{Cl}$ sample.

(ii) 20 year data set: A non-standard solar model doesn't fit the full data much worse or much better than the 3 year data. The disagreement with the complete weighted data sample, allowing only the B flux to be reduced (in this case to $0.1 \mathrm{SSM} !)$, is still at $\approx 4.5 \sigma$. Now however that now the disagreement with the unweighted average rate (requiring a flux reduction to $0.15 \mathrm{SSM}$ ) is comparably bad. Allowing the Be flux to change as well reduces the disagreement, but the fit to the unweighted average in this case is at best only marginal (99\% confidence level). The procedure of [3] decreases the goodness-of-fit dramatically, with the best fit (at $20 \%$ of the SSM boron flux) ruled out at $>5 \sigma$.

The MSW model fit to the 20 year data is shown in figures 3 and 4 . 
Notice the line of best fit is shifted slightly from the Bahcall and Bethe line due to the inclusion of the latest Homestake data but the fit is still good at the $95 \%$ confidence level. The fits to the weighted and unweighted averages are good (better than 68\%) as one might expect. If the method of [3] is used to compute the $\chi^{2}$, thus taking account of the Poisson statistics of the low points, the best fit is only acceptable at the $\approx 5 \sigma$ level! The fact that both the non-standard solar model and MSW fits, in which the prediction is a constant, are worse using the method of [3] than using a normal $\chi^{2}$ procedure suggests that this latter method is much more sensitive to "jitter" in the data.

Since it is perhaps the simplest and most elegant of the proposed neutrino based "solutions" to the solar neutrino problem we feel the MSW model deserves a closer inspection. In this regard we have developed a new way of presenting the comparison between theory and observation. For the 680 $\left(\Delta m^{2}, \sin ^{2} 2 \theta\right)$ parameter pairs we calculated in our study, we display in figure 5 a plot of the MSW predictions for Homestake vs Kamiokande. While $a$ priori one might expect such a plot to "fill" much of the plane, one can see that the allowed region is in fact a narrow band passing from bottom left to top right. This behaviour is due to the fact that high energy ${ }^{8} B$ electron neutrinos make up most of the signal for both detectors, leading to a strong correlation in the signals for an energy dependent $\nu_{e}$ flux reduction. (We thus expect that adding the neglected contributions from ${ }^{15} \mathrm{O}$ and hep neutrinos to the Homestake signal will broaden this band slightly.) Still the narrowness of the band is a surprising indication of the strong constraints on the predictions of the MSW solution. Also shown in figure 5 are the averages of the actual rates seen in the detectors. In this way one can obtain a clear and immediate graphical picture of how well the MSW solution as a whole can reproduce the observed averages. As can be seen, the fair overlap between (the constrained) theoretical phase space and the observations is suggestive.

The low points in the pre-1987 sample can be well accommodated, as 
has been previously noticed, by a time varying neutrino signal. In addition, as stressed earlier, resonant spin-flavor transitions also allow "arbitrary" Kamiokande time variation for a given variability in the $\mathrm{Cl}$ data. As expected, therefore, we find that the complete data sample can be well fit over a wide range of parameter space by a time varying magnetic field coupled with a large neutrino transition magnetic moment. Shown in figure 6 (a) and (b) are the regions of mass-mixing angle space allowed at the 68 and 95\% confidence levels when the magnetic field time variation is fixed at the value which provides the minimal $\chi^{2}$ fit to the data for a (a) cosine or (b) sawtooth time dependence. (The actual region of parameter space allowed in this case is a 4 dimensional space in mass, mixing angle, and magnetic field time variation - difficult to draw, but whose boundary in the extreme limit of zero magnetic field splitting would reduce to the MSW plot already presented.) The cosine fit to the data at this optimum magnetic field value is obviously better than the zero field MSW fit, while the sawtooth fit is even broader, and slightly better than the cosine fit at the optimum magnetic field value.

The apparent jitter and/or the occurrence of anomalously low data points in the $\mathrm{Cl}$ data sample, which dominates over the Kamiokande sample in the 20 year fits (by about 4 to 1 in the $\chi^{2}$ determinations), cannot be dismissed based purely on statistical grounds alone. We have investigated whether one might be forced to ignore or rescale the error bars in order to reduce this effect by examining the variance of both the weighted and unweighted $\mathrm{Cl} 20$ year samples. The mean value of the of the $\mathrm{Cl}$ signal for the complete 20 year weighted sample is $1.70 \pm .22 \mathrm{SNU}$. This is significantly smaller than the unweighted average of $2.21 \pm .24$ SNU. Nevertheless, the $\chi^{2}$ per degree of freedom for this weighted average is 1.07. This indicates that there is no necessity to rescale errors to account for the variance of the sample from the mean. Alternatively, the unweighted sample has a mean variance per point of $1.7 \mathrm{SNU}$. This is comparable to the error per point in the weighted sample, 
indicating again that there is no evidence that the errors are skewed in any way.

Finally we stress a somewhat non-intuitive result. In the 20 year sample, the $\mathrm{Cl}$ data clearly dominates in any fit. One may feel that comparing model predictions to average values may alleviate this problem by treating the two data sets with equal weight. However, the relative errors determined for the Homestake mean values are small enough so that the Homestake result dominates the fit to average values (weighted or unweighted) more than it does a fit to the complete sample. Thus, if the $\mathrm{Cl}$ data is suspect, for any reason, using average values rather than the full data set will only exacerbate this problem.

One way in which we might hope to proceed further in distinguishing between models is to examine the predictions for the Ga solar neutrino experiments (SAGE and GALLEX collaborations) which are currently beginning to run. Estimates of gallium rates predicted by the models we have considered are summarized in the last two columns of Table 2. For a given model, we have computed the range of neutrino rates that would be seen in a Ga-based detector for the region of parameter space not already excluded at the $68 \%$ and $95 \%$ confidence levels by the present Homestake and Kamiokande data. The time dependence of the predicted Gallium rates for the time-dependent models varied widely (including no significant time variation) for equally allowed parameter sets. Thus measuring the time dependence of the rates in Gallium detectors might help further constrain these models, although if uncertainties in the data are on the same order as the $\mathrm{Cl}$ data, a clear measurement of time dependence is unlikely in the short term. Moreover, an observation of no time variation in the Gallium detectors would once again not provide definitive evidence against time variation in the $\mathrm{Cl}$ signal. In the context of neutrino based models then the SAGE result, (20 \pm 38$)$ SNU, is perhaps the least enlightening result one could obtain from a theoretical point of view. 
Kamiokande itself now provides another constraint on resonant spin-flavor conversion models. Electron neutrinos can be converted to electron antineutrinos in the sun, and these contribute to the isotropic background signal in the Kamiokande detector. Thus, the flat background of isotropic events seen by the Kamiokande detector can place a limit on the flux of electron anti-neutrinos [9]. Although a careful analysis of the data in this regard has not yet been performed, estimates of the flux of electron anti-neutrinos for neutrino energies greater than or equal to $10.6 \mathrm{MeV}$ for the time period June 1988 through April 1989 are less than approximately 10\% of the expected electron neutrino flux predicted by the SSM [19]. For the models discussed in this paper, the predicted electron anti-neutrino fluxes ranged from 0 to $30 \%$ of the SSM $\nu_{e}$ flux. Figure 7 outlines regions of parameter space excluded for various flux limits, for Zeeman energies of $2.0 \times 10^{-10}$ and $5.0 \times 10^{-10} \mu \mathrm{BkG}$ respectively. (Indicative of average and peak Zeeman energy values which appear in the best fit solutions.) Note that some regions favored by the time varying models are eliminated by the $10 \%$-of-background cut, but none of the time varying models are completely eliminated on the basis of this constraint alone. As the energy threshold for the Kamiokande background subtraction is reduced, more of the parameter space for magnetic moment induced oscillations can be probed. However, it is worth noting that our results suggest that none of the present "allowed regions" for the time varying models would be eliminated even if a background cut at the $5 \%$ level were made. It is possible that the SNO heavy water detector may eventually be able to distinguish the antineutrino signal more clearly from the neutrino signal, and thus could further improve these bounds.

\section{Results}

For convenience we summarize the above analysis and restate the main results: 
1. Non-standard solar models which result in a reduced boron flux are ruled out, for the concurrent weighted data sample, at the $4 \sigma$ confidence level. This limit is basically unchanged when the rest of the $\mathrm{Cl}$ data is taken into account, though the required flux reduction is more extreme. If the unweighted $\mathrm{Cl}$ average signal is utilized instead, this simplest nonstandard solar model fits at the $98 \%$ confidence level for the concurrent data sample. In this case, however, the fit to the unweighted average of the full 20 year sample is incompatible at the $\approx 4-5 \sigma$ level, due to the low long-term Homestake average. The SAGE results now also appear to argue against this possibility.

2. The MSW neutrino mass solution of the solar neutrino model over much of the range claimed by Bahcall and Bethe fits the concurrent and 20yr weighted data at only the $95 \%$ confidence level. We have no statistical evidence that the error bars in the $\mathrm{Cl}$ data are anomalous, but if the unweighted mean is utilized instead, the MSW fits improve significantly. This suggests the jitter in the Homestake data may be the cause of the higher $\chi^{2} /$ dof. On a Homestake vs. Kamiokande plot the MSW prediction appears as a thin band which overlaps the averaged data. In this way, the agreement between theory and averaged data is more easily pictured.

3. Models with resonant spin-flavor conversion due to a varying magnetic field in the sun fit the data with a confidence level which is at least comparable to the MSW fits - even for the 3 year concurrent sample in which no time variation in the Kamiokande signal is obvious. As expected, the time-varying models provide acceptable fits to the complete weighted data set much more broadly than the MSW models do, and in the case of a sawtooth time-dependence the best fit is also greatly improved. The maximum Zeeman splitting needed in these cases is rather large, of order $2-5 \times 10^{-10} \mu B \mathrm{kG}$. 
4. Most neutrino based solutions to the solar neutrino problem not excluded at the $95 \%$ confidence level predict roughly comparable rates in Ga, between 5-65 SNU. Non-standard solar models which are not excluded predict rates greater than 90 SNU. Hence, Ga can decisively rule out non-standard solar models, but cannot distinguish well between neutrino based solutions. Acceptable time-varying models predict a wide range of possible time variation in Gallium, including almost no observable variation.

5. Kamiokande can restrict the allowed parameter range for spin-flavor conversion models, and already rules out $\Delta m^{2}$ in the range $10^{-8}-$ $10^{-7} \mathrm{eV}^{2}$, for mixing angles greater than $\sin ^{2}(2 \theta) \sim 0.3$. This limit comes from the isotropic background in the experiment and will improve with time. The SNO detector might improve these further.

\section{Conclusions}

The Kamiokande experiment can provide a useful check on the Homestake experiment, and the combined data from both experiments during their concurrent running is consistent with a wide variety of models. Unfortunately, however, the specifics of which model and what parameters appear to be favored depend upon how one treats the data, so that no categorical conclusions can yet be made.

Future experiments at Kamiokande and with Ga may not allow much finer distinctions between neutrino-based models to be made, but they could definitively rule out non-standard solar model based solutions of the solar neutrino problem. At this point 20 years of experiments have at least firmly established the existence of the solar neutrino problem and pointed to new

microphysics as the likely solution. To gain the information necessary to completely resolve this issue it will be necessary to measure the solar neutrino spectrum itself. If neutrino mixing is indeed the cause of the solar 
neutrino problem then a knowledge of which energies are most suppressed would give us a better handle on the underlying mechanism and parameters (for example in simple MSW mixing, in the regions considered here, lowering $\Delta m^{2}$ for a given mixing angle lowers the threshold energy below which $\nu_{e} \rightarrow \nu_{x}$ conversion takes place).

Experiments with this goal in mind (i.e. 24, 25]) are important to pursue. In this way a new window on physics at scales beyond those accessible at present accelerators may be fully explored.

We thank Ken Lande for providing us with the complete sets of Chlorine neutrino data and for useful discussions on both the $\mathrm{Cl}$ and $\mathrm{Ga}$ experiments, and M. Smith for informing us of the work of Filippone. We also thank C. Baltay for useful discussions, P. Langacker for helpful advice on error handling, and D. Gelernter and D. Kaminsky of the Linda group of the Department of Computer Science at Yale for running our evolution code on their complex. 


\section{References}

[1] J. N. Bahcall, "Neutrino Astrophysics", Cambridge University Press (1989); R. Davis, Jr., in "Proceedings of the Seventh Workshop on Grand Unification", Toyama, 1986, p.237, ed J. Arafune, World Scientific, (1986)

[2] K. Hirata et al., Phys. Rev. Lett. 63 (1989) 16; Phys. Rev. Lett. 65 (1990) 1297

[3] B.W. Filippone and P. Vogel, Phys. Lett. B246 (1990) 546

[4] T. Cleveland, Nucl. Instr. Meth. 214 (1983) 451

[5] S.P. Mikheyev, A. Yu. Smirnov, Sov. J. Nucl. Phys. 42 (1985) 913; L. Wolfenstein, Phys. Rev. D17 (1987) 2369

[6] J.N. Bahcall and H.A. Bethe, Phys. Rev. Lett. 65 (1990) 2233

[7] M.B.Voloshin and M.I. Vysotsky, ITEP Report No. 1 (1986); L.B. Okun, Sov. J. Nucl. Phys. 44 (1986) 546; L.B. Okun, M.B. Voloshin, and M.I. Vysotsky, Sov. J. Nucl. Phys. 44 (1986) 440; L.B. Okun, M.B. Voloshin, and M.I. Vysotsky, Sov. Phys. JETP 64 (1986) 446; A. Cisneros, Astrophys. Space Sci. 10 (1981) 87

[8] C.S. Lim and W.J. Marciano, Phys. Rev. D37 (1988) 1368

[9] C.S. Lim et al., Phys. Lett. B243 (1990) 389

[10] see S.L. Glashow and L.M. Krauss, Phys. Lett. B190 (1987) 199

[11] J.N. Bahcall and W. H. Press, Ap. J. 370 (1991) 730

[12] L.M.Krauss, Nature 348 (1990) 403 
[13] J.W. Bieber, D. Seckel, T. Stanev and G. Steigman, Nature 348 (1990) 408

[14] G. Raffelt, Phys. Rev. Lett. 64 (1990) 2856

[15] W.H. Press, B. P. Flannery, S. A. Teukolsky, W.T. Vetterling, "Numerical Recipes", Cambridge University Press, (1986)

[16] S.P. Rosen and J.M. Gelb, Phys. Rev. D34 (1986) 969

[17] L.M. Krauss, Nature 329 (1987) 689

[18] M. Nakahata, Ph.D Thesis, Search for ${ }^{8} B$ neutrinos at KAMIOKANDEII, ICEPP Preprint UT-ICEPP-88-1

[19] R. Barbieri et al., Phys. Lett. B259 (1991) 119

[20] W. Marciano, Nucl. Phys. B11 (1989) 5

[21] L.M. Krauss, Nature 355 (1992) 399

[22] A.I. Abazov et al., Phys. Rev. Lett. 67 (1991) 3332

[23] Y. Elsworth et. al., Nature 347 (1990) 536

[24] B. Cabrera, L.M. Krauss, F. Wilczek, Phys. Rev. Lett. 55 (1985) 25

[25] L.M. Krauss, F. Wilczek, Phys. Rev. Lett. 55 (1985) 122; see also J. Bahcall, IAS preprint 1991 


\begin{tabular}{llc} 
Experiment & Averaging Method & Average \\
\hline Kamiokande: & & $0.4600 \pm 0.0781$ \\
Homestake: & & \\
& 20 year weighted: & $0.2153 \pm 0.0284$ \\
& 20 year unweighted: & $0.2799 \pm 0.0309$ \\
& concurrent weighted: & $0.2475 \pm 0.0436$ \\
& concurrent unweighted: & $0.3602 \pm 0.0528$
\end{tabular}

Table 1: Average values for solar neutrino data 


\begin{tabular}{|c|c|c|c|c|}
\hline Model & $\chi^{2}$ (d.f.) & Parameters* & $\mathrm{Ga}(68 \%)$ & $\mathrm{Ga}(95 \%)$ \\
\hline \multicolumn{5}{|l|}{ Concurrent Data: } \\
\hline MSW & $32.6(23)$ & $1.58,0.25,-,-$ & & $5-56$ \\
\hline Cosine & $30.9(21)$ & $1.26,0.10,2.3,2.3$ & & $5-66$ \\
\hline Sawtooth (6.65) & $31.7(21)$ & $0.25,0.20,2.0,2.0$ & & $5-66$ \\
\hline Sawtooth (8.05) & $31.0(21)$ & $0.16,0.45,2.4,2.4$ & & $5-66$ \\
\hline $\operatorname{Cos}(20 y r)$ & $31.7(23)$ & $1.58,0.20$ & & \\
\hline Saw (20yr-6.65) & $32.1(23)$ & $1.58,0.15$ & & \\
\hline Saw (20yr-8.05) & $31.4(23)$ & $1.26,0.10$ & & \\
\hline \multicolumn{5}{|c|}{ Concurrent Data (averages): } \\
\hline MSW (weighted) & $0.76(0)$ & $1.26,0.35,-,-$ & $6-56$ & $5-56$ \\
\hline MSW (unweighted) & $.002(0)$ & $0.79,0.90,-,-$ & $6-57$ & $6-57$ \\
\hline \multicolumn{5}{|l|}{ All Data: } \\
\hline MSW & $101(93)$ & $2.51,0.20,-,-$ & & $4-58$ \\
\hline Cosine & $99.7(91)$ & $3.16,0.15,1.1,1.1$ & $8-12$ & $4-58$ \\
\hline Sawtooth (6.65) & $97.8(91)$ & $1.26,0.10,1.8,1.8$ & $7-20$ & $5-66$ \\
\hline Sawtooth (8.05) & $97.4(91)$ & $1.26,0.05,2.0,2.0$ & $5-27$ & $5-66$ \\
\hline \multicolumn{5}{|c|}{ All Data (averages): } \\
\hline MSW (weighted) & $1.64(0)$ & $5.01,0.10,-,-$ & $5-20$ & $5-55$ \\
\hline MSW (unweighted) & $0.15(0)$ & $2.51,0.04,-,-$ & $6-56$ & $6-56$ \\
\hline
\end{tabular}

${ }^{*}$ Parameters: $\Delta m^{2} / 10^{-7} e V^{2}, \sin ^{2}(2 \theta), A, B\left(/ 10^{-10} \mu B \mathrm{kG}\right)$, for Zeeman en$\operatorname{ergy}=A+B[\cos (t)$ or $\operatorname{saw}(t)]$

Table 2: Neutrino Data $\chi^{2}$ Fits and Ga Predictions 


\begin{tabular}{lccc} 
Model & $\chi^{2}$ & Flux reduction & Ga \\
\hline Concurrent Data: & \multicolumn{3}{c}{} \\
B & 67.5 & 0.25 of SSM & 122 \\
B+Be & 49.4 & 0.30 of SSM & 98 \\
Concurrent Data & (averages): & \\
B (weighted) & 20.3 & 0.18 of SSM & 121 \\
B+Be (weighted) & 9.74 & 0.25 of SSM & 96 \\
B (unweighted) & 7.73 & 0.30 of SSM & 122 \\
B+Be (unweighted) & 2.78 & 0.36 of SSM & 101 \\
All Data: & & & \\
B & 166 & 0.09 of SSM & 119 \\
B+Be & 131 & 0.20 of SSM & 93
\end{tabular}

All Data (averages):

$\begin{array}{lccc}\text { B (weighted) } & 30.4 & 0.07 \text { of SSM } & 119 \\ \text { B+Be (weighted) } & 14.6 & 0.18 \text { of SSM } & 93 \\ \text { B (unweighted) } & 20.0 & 0.15 \text { of SSM } & 120 \\ \text { B+Be (unweighted) } & 8.63 & 0.25 \text { of SSM } & 96\end{array}$

Table 3: Non standard solar model $\chi^{2}$ fits and Ga predictions 


\section{Figures}

1. Shown in (a) is the complete Homestake, and Kamiokande data set used in this analysis, with neutrino signal shown as a fraction of that predicted in the Standard Solar Model. Error bars for the $\mathrm{Cl}$ data are discussed in the text. In (b) the subset of the sample containing the data obtained concurrently by the two detectors is shown.

2. Those regions in the MSW parameter space (mass-squared difference and mixing angle) which are allowed by the 3 year concurrent data sample at the $95 \%$ confidence levels based on a comparison to (a) all the weighted concurrent data, and (b) the unweighted averages of the two concurrent data sets, are shown. The line shows the solar neutrino problem "solution" described by Bahcall and Bethe.

3. Those regions in the MSW parameter space (mass-squared difference and mixing angle) which are allowed by the full 20 year weighted data.

4. Same as the last figure, except based on (a) the weighted average signals, (b) the unweighted average signals.

5. MSW predictions for Homestake and Kamiokande experiments and experimental rates.

6. Those regions in $\Delta m^{2}-\sin ^{2} 2 \theta$ space which are allowed at the 68 and 95\% confidence levels for non-zero transition magnetic moments based on the 20 year weighted data sample, when the Zeeman energy is fixed to its "best fit" value, with time dependence:

(a) $\left(1.1 \times 10^{-10}+1.1 \times 10^{-10} \cos (f+k t)\right) \mu B \mathrm{kG}$,

(b) $\left(2 \times 10^{-10}+2 \times 10^{-10} \mathrm{saw}(t, \tau=8.05)\right) \mu B \mathrm{kG}$.

7. The predicted electron anti-neutrino signal in Kamiokande as a fraction of the observed background for incident anti-neutrinos of energy > 
$10.6 \mathrm{MeV}$, for resonant spin conversion models, if the Zeeman energy in the sun has value: (a) $2 \times 10^{-10} \mu \mathrm{BkG}$, (b) $5 \times 10^{-10} \mu \mathrm{BkG}$, is shown as a function of $\Delta m^{2}$ and $\sin ^{2}(2 \theta)$. 